\title{
Social Isolation During Adolescence Strengthens Retention of Fear Memories and Facilitates Induction of Late-Phase Long-Term Potentiation
}

\author{
Ji-Hong Liu • Qiang-Long You • Mei-Dan Wei • \\ Qian Wang • Zheng-Yi Luo • Song Lin • Lang Huang • \\ Shu-JI Li • Xiao-Wen Li • Tian-Ming Gao
}

Received: 3 August 2014 / Accepted: 28 September 2014 / Published online: 28 October 2014

(C) The Author(s) 2014. This article is published with open access at Springerlink.com

\begin{abstract}
Social isolation during the vulnerable period of adolescence produces emotional dysregulation that often manifests as abnormal behavior in adulthood. The enduring consequence of isolation might be caused by a weakened ability to forget unpleasant memories. However, it remains unclear whether isolation affects unpleasant memories. To address this, we used a model of associative learning to induce the fear memories and evaluated the influence of isolation mice during adolescence on the subsequent retention of fear memories and its underlying cellular mechanisms. Following adolescent social isolation, we found that mice decreased their social interaction time and had an increase in anxiety-related behavior. Interestingly, when we assessed memory retention, we found that isolated mice were unable to forget aversive memories when tested 4 weeks after the original event. Consistent with this, we observed that a single train of highfrequency stimulation (HFS) enabled a late-phase long-term potentiation (L-LTP) in the hippocampal CA1 region of isolated mice, whereas only an early-phase LTP was observed
\end{abstract}

Electronic supplementary material The online version of this article (doi:10.1007/s12035-014-8917-0) contains supplementary material, which is available to authorized users.

J.-H. Liu · Q.-L. You • Q. Wang · Z.-Y. Luo · S. Lin · L. Huang · S.-J. Li $\cdot$ X.-W. Li $\cdot$ T.-M. Gao

State Key Laboratory of Organ Failure Research, Department of Neurobiology, School of Basic Medical Sciences, Southern Medical University, Guangzhou 510515, China

J.-H. Liu • Q.-L. You • Q. Wang • Z.-Y. Luo • S. Lin • L. Huang • S.-J. Li $\cdot$ X.-W. Li $\cdot$ T.-M. Gao $(\triangle)$

Key Laboratory of Psychiatric Disorders of Guangdong Province, Southern Medical University, Guangzhou 510515, China

e-mail: tgao@smu.edu.cn

M.-D. Wei

Department of Pharmacy, The Third Affiliated Hospital of Southern Medical University, Guangzhou 510630, China with the same stimulation in the control mice. Social isolation during adolescence also increased brain-derived neurotrophic factor (BDNF) expression in the hippocampus, and application of a tropomyosin-related kinase $\mathrm{B}$ (TrkB) receptor inhibitor ameliorated the facilitated L-LTP seen after isolation. Together, our results suggest that adolescent isolation may result in mental disorders during adulthood and that this may stem from an inability to forget the unpleasant memories via BDNF-mediated synaptic plasticity. These findings may give us a new strategy to prevent mental disorders caused by persistent unpleasant memories.

Keywords Social isolation $\cdot$ Fear memory $\cdot$ Long-term potentiation $\cdot$ BDNF $\cdot$ TrkB receptor

\section{Introduction}

The management of fear memories is important when treating mental health disorders such as posttraumatic stress $[1,2]$. In fact, fear memories can also be problematic for individuals who do not meet the diagnostic criteria for a mental health disorder. The rate at which unpleasant memories diminish over time shows interindividual differences: For some, these memories diminish quickly, while others can continue to be affected by these memories for years or even decades [3]. It is therefore crucial to understand the mechanisms underlying these differences since the retention of disturbing memories can significantly disrupt a person's mental, physical, and social well-being. A behavioral paradigm appropriate for investigating fear memory is Pavlovian fear conditioning. In this paradigm, organisms learn to predict aversive events [4]. In this form of learning, an aversive stimulus (e.g., an electrical shock, loud noise, or unpleasant odor) is associated with a neutral context (e.g., a room) or stimulus (e.g., a tone), 
resulting in the expression of fear responses to the originally neutral stimulus or context.

Adolescence is a transitional stage of physical and psychological human development that generally occurs from puberty to legal adulthood $[5,6]$. During this time, an individual's social relationships are fundamental to emotional fulfillment, behavioral adjustment, and cognitive function. Previous studies have consistently demonstrated that social isolation predicts morbidity and mortality from cancer, cardiovascular disease, and a host of other diseases [7]. Moreover, social isolation and neglect during adolescence can influence adult cognitive function and social interactions [8-10]. So far, several studies have investigated that social isolation impaired the spatial memory [11-16].

The ability to alter emotional responses is a critical component of normal adaptive behavior and is often impaired in psychological disorders [17]. For example, some forms of anxiety disorders are thought to involve dysfunction in the neural systems underlying the extinction of fear memory [18]. Therefore, we hypothesized that social isolation during adolescence could lead to disruptions in mental health and that the underlying cause of these impairments could result from the inability to forget unpleasant memories. In addition, there are reports showing that social isolation during the late adolescence and adulthoods induces the fear memory deficit [19-23]. Even so, isolation during the early adolescence may have a different consequence and may involve different mechanism, because impaired social behaviors are found only in the mouse isolated starting from P21 but not from P35 [8]. Therefore, we set about to investigate whether social isolation during early adolescence influences fear memories and its mechanisms.

Therefore, the aim of this study was to evaluate the implications of adolescent social isolation on fear memory retention and its underlying cellular mechanisms (i.e., late-phase longterm potentiation [L-LTP]) during adulthood. Moreover, since many studies have demonstrated an intimate relationship between brain-derived neurotrophic factor (BDNF) and L-LTP [24-28], we also studied whether BDNF was involved in this phenomenon.

\section{Materials and Methods}

\section{Subjects}

Adult male C57 BL/6 mice were housed in standard laboratory cages (four to five per cage) on a 12-h light/dark cycle (lights on at 8:00 A.M.) in a temperature-controlled room (21$25^{\circ} \mathrm{C}$ ). Mice were housed with free access to food and water. At postnatal days 21-22, littermate pups were housed either individually or in groups of 3-5 mice per cage. While isolated animals could hear and smell other animals within the housing facility, there was no physical interaction with other mice. Additionally, the number of investigators handling the isolated animals during weekly cage changes was kept to a minimum [29]. Animals were isolated for 4 weeks before the initiation of any experimental procedure. Behavioral testing was performed during the light cycle between 10:00 A.M. and 5:00 P.M. All procedures were conducted in accordance with the Chinese Council on Animal Care Guidelines [30], and efforts were made to minimize animal suffering and to reduce the number of animals used.

\section{Drugs}

Emetine dihydrochloride hydrate (Emetine; Sigma-Aldrich, USA) was dissolved in distilled water. K-252a (SigmaAldrich, USA) was dissolved in dimethyl sulfoxide (DMSO), and the concentration of DMSO (Sigma) used for all solutions was less than $0.1 \%$. All other chemicals were purchased from Sigma-Aldrich. Dose selections for these drugs were based on both pilot and previously published studies [31, 32].

\section{Open-Field Test}

The open-field test was performed in a rectangular chamber $(60 \times 60 \times 40 \mathrm{~cm})$ composed of gray polyvinyl chloride; the center area of which was illuminated by $25-\mathrm{W}$ halogen bulbs (200 $\mathrm{cm}$ above the field). Mice were gently placed into the testing chamber for a 5-min recording period, which was monitored by an automated video tracking system. Images of the paths traveled in those 5 min were automatically calculated using the DigBehv animal behavior analysis program.

\section{Social Interaction Test}

This behavioral test was conducted in darkness. Mice were placed in a new area with a small, empty cage at one end. Baseline movement was tracked for $2.5 \mathrm{~min}$ and was then recorded for $2.5 \mathrm{~min}$ in the presence of a caged aggressor male. The duration each mouse spent in a predefined interaction zone and other measures were obtained using Ethovision XT (Noldus, USA) software. After each trial, the apparatus was cleaned with a solution of $70 \%$ ethanol in water to remove olfactory cues.

\section{Novelty Suppressed-Feeding Test}

After $24 \mathrm{~h}$ of food (but not water) deprivation, mice were placed into the testing box. One single pellet of food was placed on a white piece of paper positioned at the center of the testing box $(50 \times 50 \times 20 \mathrm{~cm})$, the floor of which was covered with $2 \mathrm{~cm}$ of thick padding. A stop watch was used to measure $5 \mathrm{~min}$, and latency was scored as the time at which mice began biting the food. Immediately after biting, mice 
were transferred to their home cage for another $5 \mathrm{~min}$, and food intake amount over this time was measured (home-cage food intake).

\section{Fear Conditioning Test}

Mice were first habituated to the behavioral room and were then allowed to freely explore the apparatus (MED-VFC-NIR-M; Med Associates) for $3 \mathrm{~min}$. During training, mice were placed in a conditioning chamber and exposed to tone-foot shock pairings (tone, $30 \mathrm{~s}$, $80 \mathrm{~dB}$; foot shock, $1 \mathrm{~s}, 0.4 \mathrm{~mA}$ ) with an interval of $80 \mathrm{~s}$ at $24 \mathrm{~h}$ after training. Mice were returned to the chamber to evaluate contextual fear learning. Mice were housed isolated during the whole fear conditioning test. Freezing during training and testing was scored using Med Associates Video-Tracking and scoring software.

\section{Electrophysiological Recordings}

This study's entire protocol is based on previous studies from our laboratory [33]. In brief, mice were decapitated, and transverse hippocampal slices $(400 \mu \mathrm{m})$ were prepared using a Vibroslice (VT 1000S; Leica) in ice-cold artificial cerebral spinal fluid (ACSF). For field potential recording, the same ACSF was used for sectioning and recording and contained (in $\mathrm{mM}$ ) $120 \mathrm{NaCl}, 2.5 \mathrm{KCl}, 1.2 \mathrm{NaH} 2 \mathrm{PO} 4$, $2.0 \mathrm{CaCl}$, $2.0 \mathrm{MgSO} 4,26 \mathrm{NaHCO}$, and 10 glucose. After sectioning, hippocampal slices were incubated for $30 \mathrm{~min}$ at $34{ }^{\circ} \mathrm{C}$ and then at room temperature $(25 \pm$ $1{ }^{\circ} \mathrm{C}$ ) for an additional 2-8 h. All solutions were saturated with $95 \% \mathrm{O}_{2} / 5 \% \mathrm{CO}_{2}(v / v)$.

Slices were placed in a recording chamber, which was superfused $(3 \mathrm{~mL} / \mathrm{min})$ with ACSF at $32-34{ }^{\circ} \mathrm{C}$. Field excitatory postsynaptic potentials (fEPSPs) were evoked in the CA1 stratum radiatum by stimulating Schaffer collaterals (SC) with a two-concentrical bipolar stimulating electrode (FHC). The evoked responses were recorded in currentclamp mode by the Axon MultiClamp 700B (Molecular Devices) amplifier with ACSF-filled glass pipettes (1$5 \mathrm{M} \Omega$ ). Test stimuli consisted of monophasic $100-\mu \mathrm{s}$ pulses of constant currents (with intensity adjusted to produce $25 \%$ of the maximum response) at a frequency of $0.033 \mathrm{~Hz}$. The strength of synaptic transmission was determined by measuring the initial (10-60\% rising phase) slope of fEPSPs. LTP was induced by one or four stimulus trains (with stimulation at test stimulus intensity) delivered at $100 \mathrm{~Hz}$, with each train having 50 pulses, and an intertrain interval of $10 \mathrm{~s}$. The level of LTP was determined at an average of 0-180 min after tetanic stimulation.

\section{ELISA}

Mice were deeply anesthetized and perfused with $40 \mathrm{~mL}$ of $\mathrm{Ca} 2^{+} / \mathrm{Mg}^{+}{ }^{+}$-free Dulbecco's phosphate-buffered saline (DPBS). CNS tissues were harvested, and the cortex, striatum, and hippocampus were dissected. Tissues were disrupted by sonication in NP-40 lysis buffer $(50 \mathrm{mM}$ Tris $\mathrm{pH}=7.4$, $150 \mathrm{mM} \mathrm{NaCl}, 1 \% \mathrm{NP}-40,0.1 \%$ Triton X-100, and $0.1 \%$ SDS) supplemented with protease inhibitors. The homogenized material was centrifuged at $20,000 \times g$ for $15 \mathrm{~min}$, and the cleared supernatant was collected. For detection of BDNF, cleared samples were treated with $1 \mathrm{~N} \mathrm{HCl}$ for $15 \mathrm{~min}$ at room temperature, followed by neutralization with $1 \mathrm{~N} \mathrm{NaOH}$. Total protein levels in CNS homogenates were determined by bicinchoninic acid (BCA) assay (Pierce). Cytokines were measured with Platinum mouse ELISA kits (eBioscience), and BDNF levels were measured using the Emax ImmunoAssay System ELISA kit (Promega) according to the manufacturer's instructions.

\section{Statistical Analyses}

The number of experimental animals is indicated by " $n$." An independent sample $t$ test or one-way analysis of variance (ANOVA) followed by the least significant difference (LSD) for post hoc comparisons was used for statistical analysis throughout the study using SPSS software (SPSS, Inc.). All data are expressed as mean \pm SEM. The statistical significance level for all tests was set at $p<0.05$.

\section{Results}

Social Isolation During Adolescence Alters Adult Social Interaction and Novelty-Suppressed Feeding

To substantiate the effects of social isolation during adolescence on adulthood, C57 mice were socially isolated following weaning or were reared in normal conditions. After 4 weeks of isolation, the following behavioral tests were performed on the experimental mice: open field, social interaction, and the novelty-suppressed feeding (which entailed food deprivation for $24 \mathrm{~h}$ prior to start of the examination). To minimize their suffering, only one batch of mice underwent all the behavioral tests. Moreover, mice were allowed to rest for 3-4 days between tests.

Figure 1a demonstrates that social isolation did not affect general locomotor activity as indicated by total path length in the open-field test $[t(18)=-1.321 ; p=0.203$; Fig. 1a]. However, we found that socially isolated adolescents spent less time interacting with other mice during adulthood when compared to control mice $[t(18)]=4.376 ; p<0.001$; Fig. 1b]. 
Fig. 1 Social isolation during adolescence alters adult social interaction and NSF. a Mice tested 4 weeks after isolation from weaning spent the same distance in the open-field test. b Isolated mice spent less time in the interaction zone when there was a target mouse than control mice. No difference was observed in the time in the interaction zone when there was no target mouse or corner zone. c, $\mathbf{d}$ In the noveltysuppressed feeding test, the latency to explore and bite the food was increased, but no difference in food intake amount was observed. Vertical bars represent the mean $\pm \mathrm{SEM}$. Asterisks indicate significant differences from the relevant controls ( $n=10$ /group, $p<0.05$, ${ }^{* *} p<0.01$, two-tailed $t$ test)
A

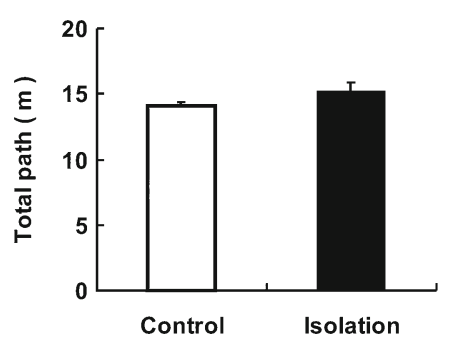

C

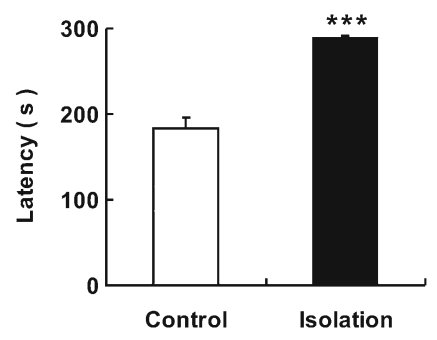

B

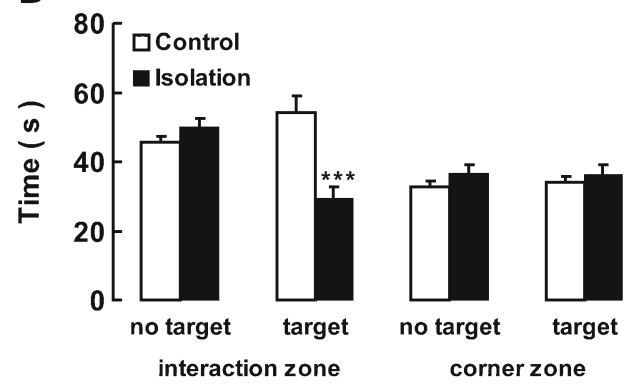

D

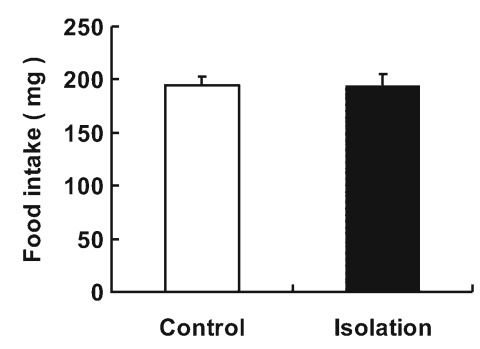

Similar results were observed in latency times for the noveltysuppressed feeding test: Social isolation increased the latency to approach and begin eating the food $[t(18)=-8.452$; $p<0.001$; Fig. $1 \mathrm{c}]$ without affecting overall food intake $[t$ $(18)=0.067 ; p=0.948$; Fig. 1d]. The above results support the hypothesis that social isolation during adolescence leads to social and mood disorders in adults.

\section{Adolescent Social Isolation Strengthens the Retention of Fear Memories}

To examine the effects of the adolescent social isolation on fear memory during adulthood, both control and isolated mice underwent hippocampal-dependent contextual fear conditioning [34]. Briefly, animals learned to fear a neutral conditioned stimulus that had been paired with an aversive unconditioned stimulus (such as foot shock) on the first day. Contextual conditioning was then measured $1,7,10,14$, and 28 days after training. We found that mice that had undergone social isolation exhibited a longer retention of fear memory (Fig. 2). Student's $t$ test indicated no significant difference in freezing during training $(t(20)=0.702, p=0.491)$ and context tested at $1(t(20)=1.343, p=0.199), 7(t(20)=0.758, p=0.457)$, and 10 $(t(20)=0.747, p=0.464)$ days between control and isolated mice, but a significant group difference in contextual freezing was found when mice were tested at $14(t(20)=-5.004$, $p<0.001)$ and $28(t(20)=-3.746, p=0.001)$ days. Compared with control mice, isolated mice displayed longer freezing times at the second and fourth weeks after training, indicating strengthened retention of fear memory. In other words, socially isolated adolescents exhibited an inability to forget the intrusive memory of the foot shock in adulthood.
Social Isolation During Adolescence Facilitates the Induction of Late-Phase Long-Term Potentiation

Hippocampus LTP is assumed to represent the cellular mechanism underlying learning and memory $[35,36]$. To investigate the cellular mechanism underlying the social isolationstrengthened fear memory, we recorded fEPSPs in the dendritic region of CA1 and compared the LTP induction between hippocampal slices taken from isolated (isolated for 4 weeks) and control animals. We first induced LTP using one train of high-frequency stimulation (HFS), which was used to induce early-phase LTP (E-LTP) [33, 35, 37-39]. Using this stimulation, we determined that there was no difference in E-LTP between hippocampal slices from isolated and control animals. However, unlike controls, whose L-LTP was absent after $1 \mathrm{~h}$ of induction, the L-LTP of slices from isolated mice was maintained for all $3 \mathrm{~h}$ (Fig. 3a). In the one-train HFSinduced L-LTP, the slope of fEPSPs was $93.37 \pm 1.99(n=6)$ and $135.62 \pm 2.01(n=6)$ in control and isolated groups, respectively $[t(10)=-47.294 ; p<0.001$; Fig. 3b]. Compared with controls, it was easier to induce L-LTP in slices obtained from isolated animals. We also performed complete inputoutput (I-O) curves at a series of increasing stimulation intensities in both control and isolated slices and observed no detectable changes in basal synaptic transmission (Fig. 3c).

We then induced LTP using four trains of HFS, which is normally used to induce L-LTP [24, 32, 39]. Using four trains, we found that not only the E-LTP was normal, but also that the L-LTP could be maintained for $3 \mathrm{~h}$ in both control and isolated slices (Fig. 3d, e). Moreover, there was no significant difference in I-O curves between control and isolated slices (Fig. 3f), indicating that their basal synaptic transmission 


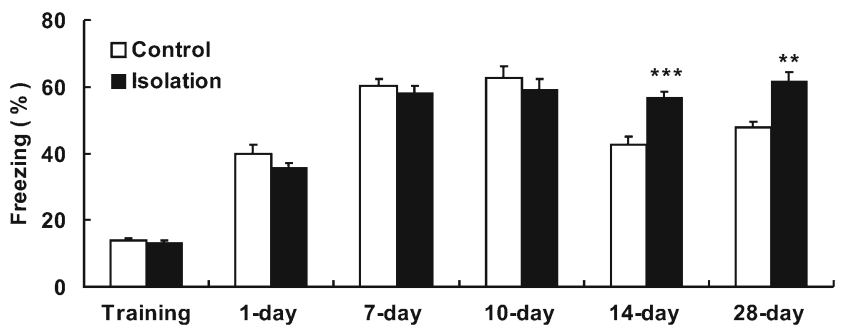

Fig. 2 Adolescent social isolation strengthens the retention of fear memories. Fear memories for contextual training 14 and 28 days after training were increased in isolated mice $(n=12)$ compared with control mice $(n=$ 10), without any changes in other days. Vertical bars represent the mean \pm SEM. Asterisks indicate significant differences from the relevant controls $(* * * p<0.001, * * p<0.01$, two-tailed)

was not changed. As L-LTP requires not only the modification of existing proteins and their trafficking at synapses, but also de novo protein synthesis [40-44], we tested whether the four trains of HFS really were induced L-LTP. In order to do this, we employed a commonly used protein synthesis inhibitor emetine $(20 \mu \mathrm{M})$ and found that L-LTP was impaired in emetine-treated compared to control groups (Fig. S1.A). In the HFS-induced L-LTP, the slope of fEPSPs was $157.01 \pm$ 2.89 and $109.81 \pm 3.46$ in control and emetine-treated groups, respectively $[t(8)=48.538 ; p<0.001$; Fig. S1.B]. Additionally, emetine did not change basal synaptic transmission (Fig. S1.C).

Taken together, our data demonstrated that social isolation during adolescence facilitated the induction of L-LTP in the hippocampus.

Adolescent Social Isolation Increases BDNF Protein Levels in the Hippocampus

Many genetic and pharmacological studies have suggested that BDNF is necessary for L-LTP [24-28, 45]. Heterozygous BDNF (+/-) knockout mice have a significant deficit in L-
Fig. 3 Social isolation during adolescence facilitates the induction of L-LTP. a, d Normalized fEPSP slope is plotted from control slices (open circles) and isolated slices (closed circles). Arrow indicates $1 * \mathrm{HFS}$ $(100 \mathrm{~Hz}, 1 \mathrm{~s})$ tetanus stimulation in $\mathrm{A}$ and $4 * \mathrm{HFS}(100 \mathrm{~Hz}, 1 \mathrm{~s}$ with 10 -s interval) in d. b, e Histogram showing average percentage of potentiation after tetanus versus baseline $(100 \%)$ at control and isolated mice. Left showed the comparing between the E-LTP (40-50 min after tetanus versus baseline) of the two groups; right showed the comparing between the L-LTP (170-180 min after tetanus vs baseline). $\mathbf{c}, \mathbf{f}$

Adolescence social isolation does not shift I-O curve. Input/output curve of fEPSP $(\mathrm{mV} / \mathrm{ms})$ versus stimulation intensity $(\mathrm{mV})$ were taken from control and isolated mice. Vertical bars represent the mean \pm SEM. Asterisks indicate significant differences from the relevant controls $(* * * p<0.001$, two-tailed)
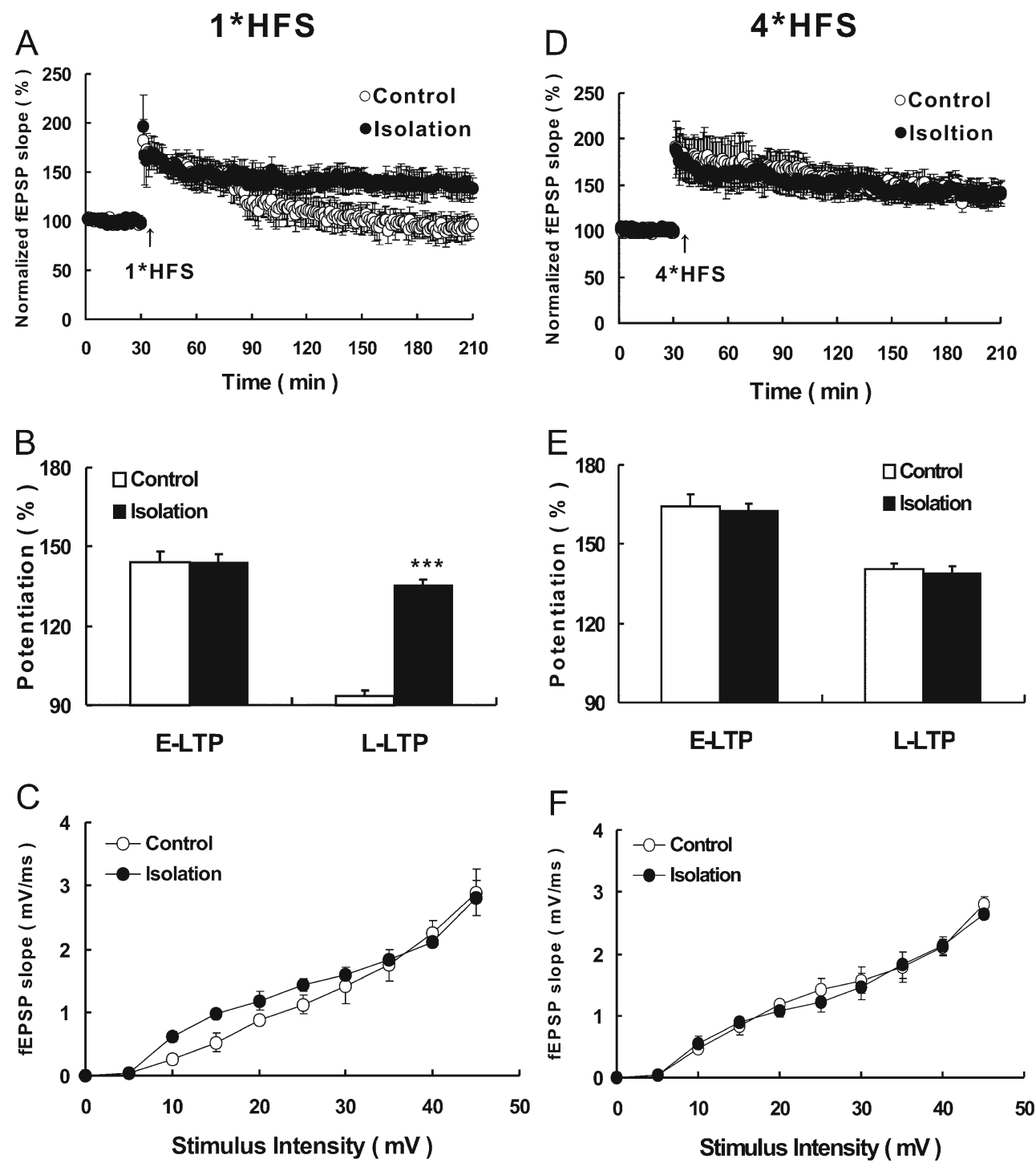
LTP [45-47]. Moreover, other studies have reported that BDNF mRNA levels increase in the hippocampal CA1 region and dentate gyrus within $2-4 \mathrm{~h}$ after application of L-LTPinducing tetanic stimulation [48-50]. These investigations collectively revealed the important role of BDNF in the induction of L-LTP. To test whether the facilitated induction of L-LTP in socially isolated mice was due to increased levels of BDNF, we examined BDNF protein levels in control and isolated mice using ELISA.

After 4 weeks of isolation, mice were decapitated, and their brains were quickly removed. We then separated the medial prefrontal cortex (mPFC), amygdala, and hippocampus from control and isolated mice and found significantly increased BDNF protein levels in the hippocampus of socially isolated mice $(50.57 \pm 1.75 \mathrm{pg} / \mathrm{mL})$ compared with the control mice $(40.81 \pm 1.58 \mathrm{pg} / \mathrm{mL})(t(4)=4.141, p=0.014$; Fig. 4). No significant difference was detected in BDNF protein levels in the $\mathrm{mPFC}$ (control, $37.96 \pm 2.37 \mathrm{pg} / \mathrm{mL}$ vs isolation, $44.52 \pm$ $3.78 \mathrm{pg} / \mathrm{mL}$ ) and amygdala (control, $53.04 \pm 2.25 \mathrm{pg} / \mathrm{mL}$ vs isolation, $55.75 \pm 3.68 \mathrm{pg} / \mathrm{mL}$ ) between the two groups.

Our results suggest that social isolation during adolescence selectively increases the level of BDNF protein in the hippocampus and that this may contribute to the enhancement of LLTP induction.

\section{K-252a Rescues Social Isolation-Facilitated L-LTP}

BDNF is a small dimeric protein that works through highaffinity binding with the receptor tyrosine kinase, tropomyosin-related kinase B (TrkB) [51, 52]. BDNF and TrkB are widely distributed across subregions of the adult hippocampus [50]. Previous studies have shown that TrkB [51] and BDNF [52] participated in modulating L-LTP. It has also been shown that K-252a can block L-LTP by inhibiting TrkB receptor [31, 53].

To further test the involvement of BDNF signaling in facilitated L-LTP, we applied the TrkB receptor inhibitor $\mathrm{K}-252 \mathrm{a}$ and found that a train of HFS could not induce L-
LTP in either isolated or control mice (Fig. 5a). The fEPSP slope was $106.35 \pm 3.17$ in the isolated group, which was similar to that of the control group (Fig. 5b). Moreover, we confirmed that K-252a treatment did not alter the basal synaptic transmission (Fig. 5c).

These data suggested a contribution of the enhanced BDNF signaling in the facilitation of L-LTP caused by social isolation.

\section{Discussion}

The major findings of this study are as follows. First, social isolation during adolescence led to decreased social interaction and increased anxiety-related behavior (as assessed by latency to approach and eat food in the novelty-suppressed feeding test). Second, social isolation strengthened the retention of fear memory and facilitated L-LTP induction in the hippocampus. Third, adolescent social isolation selectively increased hippocampal BDNF protein levels, which facilitated L-LTP via the TrkB receptor. Altogether, our results suggest that social isolation during adolescence disturbs mental health in adulthood, which may result from a disability to forget unpleasant memories via BDNF-mediated synaptic plasticity.

In rodents, it is generally agreed upon that adolescence is the period from weaning to early adulthood and is often marked by discrete developmental stages that have been described by numerous neurobiological and behavioral studies [54]. Clinical and preclinical data implicate that adverse earlylife experiences lead to later development of psychiatric and substance abuse disorders [55-57]. Although other covariates should be taken into account, approximately $80 \%$ of young adults who report early-life neglect or abuse are diagnosed with at least one psychiatric illness, including anxiety, affective, schizoaffective, or behavioral disorders [55, 56, 58-62]. To verify this phenomenon, we isolated mice just after weaning and tested the impact of isolation on their behaviors during adulthood, because the age from P21 to P35 is a crucial period for disordered behaviors during adulthood [8]. Our

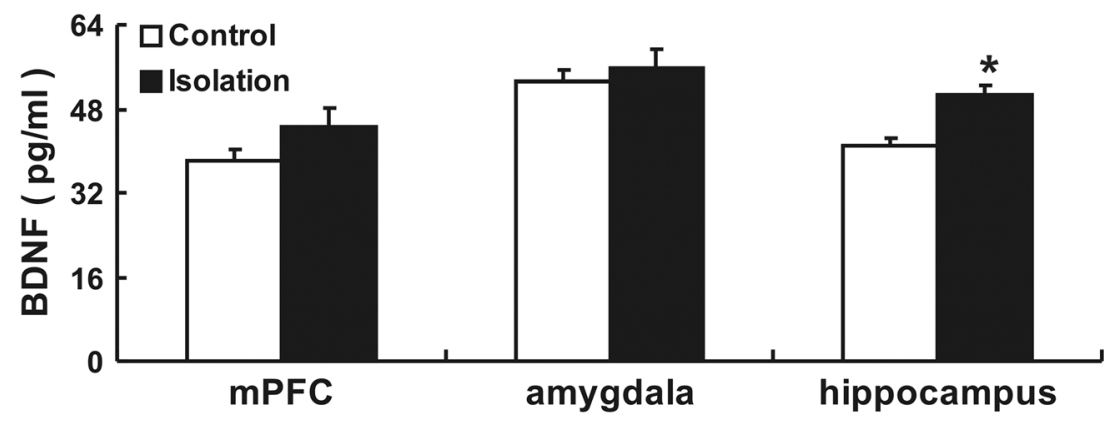

Fig. 4 Adolescence social isolation increases the BDNF protein level in hippocampus. Histogram showed BDNF protein level detected by ELISA from $\mathrm{mPFC}$, amygdala, and hippocampus of control and isolated mice. Difference was found only in hippocampus of isolated mice $(n=3)$

compared with the control mice $(n=3)$. Vertical bars represent the mean \pm SEM. Asterisks indicate significant differences from the relevant controls $\left({ }^{*} p<0.05\right.$, two-tailed) 


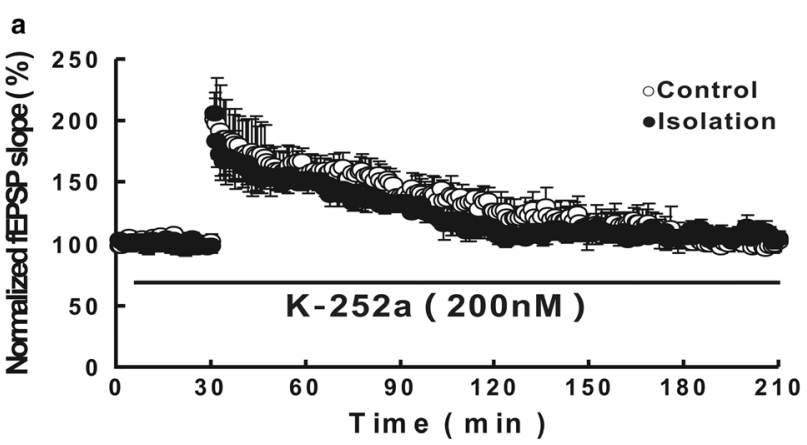

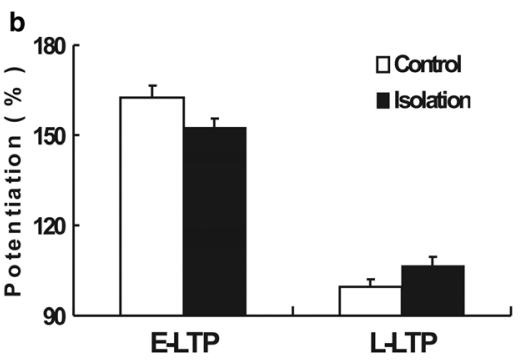

Fig. 5 Acutely applied K-252a can rescue the facilitation of L-LTP in isolated mice. a Normalized fEPSP slope is plotted from control slices (open circles) and isolated slices (closed circles), and K-252a (200 nM) was applied starting $20 \mathrm{~min}$ before tetanus (closed circles). b Histogram showed average percentage of potentiation after tetanus versus baseline $(100 \%)$ at control and isolated slices. Left showed the comparing

results supported the idea that social isolation during adolescence can cause some mental disorders, and this was reflected in the social interaction and NSF test.

As already mentioned, fear conditioning is an effective behavioral paradigm to arouse unpleasant, intrusive memories. Thus, we next evaluated the retention of fear memories in isolated versus normally housed mice. As expected, isolated mice displayed longer freezing time, indicating that they did not forget the fear of a foot shock when compared with control mice. Our study also showed that there was no obvious difference in the freezing time between control and isolation group until 2 weeks. This is not consistent with other findings. For example, Okada et al. [19] found that social isolation stress impaired consolidation processes of fear memory tested at $24 \mathrm{~h}$ after fear conditioning. Besides, fear learning was also disrupted after isolation [20]. These controversies may be interrupted by a different starting time for isolation. In our study, isolation started immediately after weaning whereas a week later in their studies. Those studies indicate that there is remarkable different consequence to the social isolation between the early and late adolescence. In support, isolation starting from P21 but not from P35 can impair social behaviors [8]. LTP is the most widely used paradigm to study cellular and molecular events underlying neuronal plasticity, and it is considered to be a model of learning and memory [36]. LTP can be separated into an early and later phase. ELTP is short lasting and is independent of new protein synthesis, whereas L-LTP requires activation of cAMP signaling

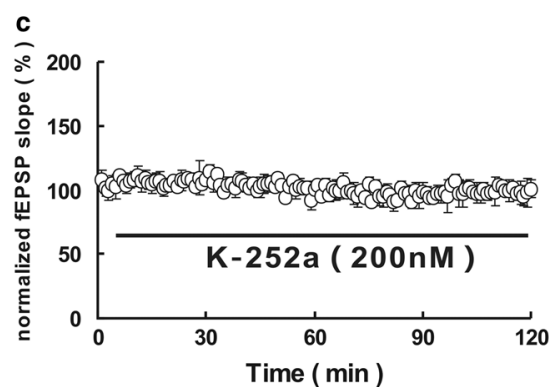

between the E-LTP (40-50 min after tetanus vs baseline) of the two groups; right showed the comparing between the L-LTP (170-180 min after tetanus vs baseline). c K-252a did not change the basal synaptic transmission. K-252a was applied $10 \mathrm{~min}$ after the beginning. Vertical bars represent the mean \pm SEM

pathway and new protein synthesis [40]. Previous studies have demonstrated that long-term social isolation of adult animals induces a reduction in the size of CA1 and a decrease in synaptic levels of polysialylated neuronal cell adhesion molecule (PSA-NCAM) in the hippocampus [14]. In the current study, we found that social isolation during adolescence can facilitate the induction of L-LTP. As has been shown throughout the literature, L-LTP can only be induced by four (or more) trains of HFS. In our study, however, one train of HFS was adequate to induce L-LTP, implying that isolation during adolescence facilitated the formation of longterm memory. Together, with the results that social isolation during adolescence might lead to certain disruptions in mental health, we hypothesized that isolated mice are unable to forget fear memories that occur during isolation. From these data, we can infer that socially isolated mice might be less capable of dealing with fear memories and that this might predispose them to developing mental disorders. This is in agreement with the fact that symptoms of major depressive disorder (MDD) often overlap with those of depressed moods [63]. It is thus likely that the inability to forget a traumatic memory might cause individuals to exhibit a depressed mood. If this were the case, then this would shed light on new ways to address relieving symptoms of MDD.

In the current study, we also found that socially isolated mice exhibited increased levels of hippocampal BDNF and that the TrkB receptor inhibitor, K-252a, can rescue the facilitation of L-LTP. These findings indicate an involvement of 
the BDNF/TrkB pathway in the late phase of LTP. One previous study showed that LTP induction evoked increases in BDNF mRNA levels in the CA1 region of the hippocampus [50]. Therefore, our data are consistent with that previous study and reinforce the idea that BDNF is involved in L-LTP. As we all known, biological action of BDNF is mediated by two receptors, the TrkB receptor and $775[52,64,65]$. BDNF is first synthesized as a precursor (proBDNF) and is cleaved to form mature BDNF (mBDNF) [66]. Mature BDNF interacts preferentially with TrkB [51], and proBDNF binds 775 with high affinity [67]. TrkB is necessary for L-LTP [45-47], while prior studies indicate that recombinant proBDNF facilitates LTD in hippocampal slices by activation of p75 [65, 68]. As this reason, we selected the TrkB receptor inhibitor to rescue isolation-induced the facilitation of L-LTP. Fear conditioning is dependent on the same brain regions that are highly susceptible to effects of stressors, including the amygdale, hippocampus, and the medial prefrontal cortex [69]. Evidence from lesion studies has shown that patients with hippocampal lesions showed retention deficits [70, 34]. Also, Karl Deisseroth et al. [71] found that hippocampal CA1 optogenetic inhibition blocked remote fear memory recall. And, in our study, we observed a longer retention time and a higher BDNF level in hippocampus in isolation group. Our results give the possibility that hippocampus but not amygdala may be related to the fear memory retention, which should be tested in the future.

In conclusion, these results demonstrate that social isolation during adolescence strengthens the retention of fear memories and facilitates the induction of L-LTP. Moreover, it is conceivable that the inability to forget an unpleasant memory might explain why some individuals develop mental disorders in their adulthood. Taken together, the findings of this study contribute to our current understanding of the negative effects of the unforgotten unpleasant things on the occurrence of mental disorders, which may help to find a new way to relieve them.

\begin{abstract}
Acknowledgments This work was partly supported by grants from the National Natural Science Foundation of China (81329003, U1201225, 31430032), the Guangzhou Science and Technology Project (7411802013939) and Program for Changjiang Scholars and Innovative Research Team in University (IRT1142).
\end{abstract}

\section{Conflict of Interest None.}

Open Access This article is distributed under the terms of the Creative Commons Attribution License which permits any use, distribution, and reproduction in any medium, provided the original author(s) and the source are credited.

\section{References}

1. Levy BJ, Anderson MC (2008) Individual differences in the suppression of unwanted memories: the executive deficit hypothesis. Acta Psychol (Amst) 127:623-635

2. van der Kolk BA, Fisler R (1995) Dissociation and the fragmentary nature of traumatic memories: overview and exploratory study. J Trauma Stress 8:505-525

3. Staten RT, Delaney KR (2010) IOM releases report on preventing mental, emotional, and behavioral disorders among young people: progress and possibilities. J Child Adolesc Psychiatr Nurs 23:118

4. Maren S (2001) Neurobiology of Pavlovian fear conditioning. Annu Rev Neurosci 24:897-931

5. Sisk CL, Foster DL (2004) The neural basis of puberty and adolescence. Nat Neurosci 7:1040-1047

6. Kulin HE, Muller J (1996) The biological aspects of puberty. Pediatr Rev 17:75-86

7. Hawkley LC, Cacioppo JT (2003) Loneliness and pathways to disease. Brain Behav Immun 17(Suppl 1):S98-105

8. Makinodan M et al (2012) A critical period for social experiencedependent oligodendrocyte maturation and myelination. Science 337:1357-1360

9. Widom CS, DuMont K, Czaja SJ (2007) A prospective investigation of major depressive disorder and comorbidity in abused and neglected children grown up. Arch Gen Psychiatry 64:49-56

10. Bos K et al (2011) Psychiatric outcomes in young children with a history of institutionalization. Harv Rev Psychiatry 19:15-24

11. Hsiao YH et al (2012) Amelioration of social isolation-triggered onset of early Alzheimer's disease-related cognitive deficit by Nacetylcysteine in a transgenic mouse model. Neurobiol Dis 45: $1111-1120$

12. Miyazaki T et al (2012) Disrupted cortical function underlies behavior dysfunction due to social isolation. J Clin Invest 122:2690-2701

13. Zhou SJ et al (2009) Preferential enhancement of working memory in mice lacking adenosine $\mathrm{A}(2 \mathrm{~A})$ receptors. Brain Res 1303:74-83

14. Pereda-Perez I et al (2013) Long-term social isolation in the adulthood results in CA1 shrinkage and cognitive impairment. Neurobiol Learn Mem 106:31-39

15. Kehoe P, Bronzino DJ (1999) Neonatal stress alters LTP in freely moving male and female adult rats. Hippocampus 9:651-658

16. Rodriguez MP et al (2005) Previous stress facilitates fear memory, attenuates GABAergic inhibition, and increases synaptic plasticity in the rat basolateral amygdala. J Neurosci 25 : 8725-8734

17. Hartley CA, Phelps EA (2010) Changing fear: the neurocircuitry of emotion regulation. Neuropsychopharmacology 35:136-146

18. Rauch SL, Shin LM, Phelps EA (2006) Neurocircuitry models of posttraumatic stress disorder and extinction: human neuroimaging research-past, present, and future. Biol Psychiatry 60:376-382

19. Okada R et al (2014) Social isolation stress-induced fear memory deficit is mediated by down-regulated neuro-signaling system and Egr-1 expression in the brain. Neurochem Res 39(5):875-82

20. Gresack JE et al (2010) Isolation rearing-induced deficits in contextual fear learning do not require $\mathrm{CRF}(2)$ receptors. Behav Brain Res 209(1):80-4

21. Morrissey MD, Mathews IZ, McCormick CM (2011) Enduring deficits in contextual and auditory fear conditioning after adolescent, not adult, social instability stress in male rats. Neurobiol Learn Mem 95(1):46-56

22. Rudy JW, Kuwagama K, Pugh CR (1999) Isolation reduces contextual but not auditory-cue fear conditioning: a role for endogenous opioids. Behav Neurosci 113(2):316-23

23. Rudy JW (1996) Postconditioning isolation disrupts contextual conditioning: an experimental analysis. Behav Neurosci 110(2):238-46 
24. Lu Y, Christian K, Lu B (2008) BDNF: a key regulator for protein synthesis-dependent LTP and long-term memory? Neurobiol Learn Mem 89:312-323

25. Bekinschtein $P$ et al (2008) BDNF is essential to promote persistence of long-term memory storage. Proc Natl Acad Sci U S A 105:2711-2716

26. Messaoudi E et al (2002) Brain-derived neurotrophic factor triggers transcription-dependent, late phase long-term potentiation in vivo. J Neurosci 22:7453-7461

27. Alonso M et al (2005) Endogenous BDNF is required for long-term memory formation in the rat parietal cortex. Learn Mem 12:504-510

28. Bekinschtein $\mathrm{P}$ et al (2007) Persistence of long-term memory storage requires a late protein synthesis- and BDNF-dependent phase in the hippocampus. Neuron 53:261-277

29. Pietropaolo $\mathrm{S}$ et al (2008) The postweaning social isolation in C57BL/6 mice: preferential vulnerability in the male sex. Psychopharmacology (Berl) 197:613-628

30. Zhu XH et al (2010) Intermittent hypoxia promotes hippocampal neurogenesis and produces antidepressant-like effects in adult rats. $\mathrm{J}$ Neurosci 30:12653-1263

31. Ji Y et al (2013) Acute and gradual increases in BDNF concentration elicit distinct signaling and functions in neurons. Nat Neurosci 13: 302-309

32. Volk LJ et al (2013) PKM-zeta is not required for hippocampal synaptic plasticity, learning and memory. Nature 493:420-423

33. Chen YJ et al (2010) ErbB4 in parvalbumin-positive interneurons is critical for neuregulin 1 regulation of long-term potentiation. Proc Natl Acad Sci U S A 107:21818-21823

34. Phillips RG, LeDoux JE (1992) Differential contribution of amygdala and hippocampus to cued and contextual fear conditioning. Behav Neurosci 106:274-285

35. Malenka RC, Nicoll RA (1999) Long-term potentiation-a decade of progress? Science 285:1870-1874

36. Bliss TV, Collingridge GL (1993) A synaptic model of memory: long-term potentiation in the hippocampus. Nature 361:31-39

37. Urban NN, Barrionuevo G (1996) Induction of hebbian and nonhebbian mossy fiber long-term potentiation by distinct patterns of high-frequency stimulation. J Neurosci 16:4293-4299

38. Blitz DM, Foster KA, Regehr WG (2004) Short-term synaptic plasticity: a comparison of two synapses. Nat Rev Neurosci 5:630-640

39. Neves G, Cooke SF, Bliss TV (2008) Synaptic plasticity, memory and the hippocampus: a neural network approach to causality. Nat Rev Neurosci 9:65-75

40. Frey U et al (1988) Anisomycin, an inhibitor of protein synthesis, blocks late phases of LTP phenomena in the hippocampal CA1 region in vitro. Brain Res 452:57-65

41. Harris KM, Fiala JC, Ostroff L (2003) Structural changes at dendritic spine synapses during long-term potentiation. Philos Trans R Soc Lond B Biol Sci 358:745-748

42. Kandel ER (2001) The molecular biology of memory storage: a dialogue between genes and synapses. Science 294:1030-1038

43. Krug M, Lossner B, Ott T (1984) Anisomycin blocks the late phase of long-term potentiation in the dentate gyrus of freely moving rats. Brain Res Bull 13:39-42

44. Yuste R, Bonhoeffer T (2001) Morphological changes in dendritic spines associated with long-term synaptic plasticity. Annu Rev Neurosci 24:1071-1089

45. Korte $\mathrm{M}$ et al (1995) Hippocampal long-term potentiation is impaired in mice lacking brain-derived neurotrophic factor. Proc Natl Acad Sci U S A 92:8856-8860

46. Pang PT et al (2004) Cleavage of proBDNF by tPA/plasmin is essential for long-term hippocampal plasticity. Science 306:487-491

47. Patterson SL et al (2001) Some forms of cAMP-mediated longlasting potentiation are associated with release of BDNF and nuclear translocation of phospho-MAP kinase. Neuron 32:123-140
48. Castren E et al (1993) The induction of LTP increases BDNF and NGF mRNA but decreases NT-3 mRNA in the dentate gyrus. Neuroreport 4:895-898

49. Dragunow M et al (1993) Brain-derived neurotrophic factor expression after long-term potentiation. Neurosci Lett 160:232-236

50. Patterson SL et al (1992) Neurotrophin expression in rat hippocampal slices: a stimulus paradigm inducing LTP in CA1 evokes increases in BDNF and NT-3 mRNAs. Neuron 9:1081-1088

51. Huang EJ, Reichardt LF (2003) Trk receptors: roles in neuronal signal transduction. Annu Rev Biochem 72:609-42

52. Lu B (2003) BDNF and activity-dependent synaptic modulation. Learn Mem 10(2):86-98

53. Bramham CR, Messaoudi E (2005) BDNF function in adult synaptic plasticity: the synaptic consolidation hypothesis. Prog Neurobiol 76: 99-125

54. Kang H et al (1997) Neurotrophins and time: different roles for TrkB signaling in hippocampal long-term potentiation. Neuron 19:653-664

55. Zhou LJ et al (2008) BDNF induces late-phase LTP of C-fiber evoked field potentials in rat spinal dorsal horn. Exp Neurol 212: 507-514

56. McCormick CM et al (2010) Investigations of HPA function and the enduring consequences of stressors in adolescence in animal models. Brain Cogn 72:73-85

57. Gutman DA, Nemeroff CB (2003) Persistent central nervous system effects of an adverse early environment: clinical and preclinical studies. Physiol Behav 79:471-478

58. Lapiz MD et al (2003) Influence of postweaning social isolation in the rat on brain development, conditioned behavior, and neurotransmission. Neurosci Behav Physiol 33:13-29

59. Fone KC, Porkess MV (2008) Behavioural and neurochemical effects of post-weaning social isolation in rodents-relevance to developmental neuropsychiatric disorders. Neurosci Biobehav Rev 32: 1087-1102

60. McFarlane A et al (2005) The impact of early life stress on psychophysiological, personality and behavioral measures in 740 nonclinical subjects. J Integr Neurosci 4:27-40

61. Espejo EP et al (2007) Stress sensitization and adolescent depressive severity as a function of childhood adversity: a link to anxiety disorders. J Abnorm Child Psychol 35:287-299

62. Heim C et al (2008) The link between childhood trauma and depression: insights from HPA axis studies in humans. Psychoneuroendocrinology 33:693-710

63. Nestler EJ et al (2002) Neurobiology of depression. Neuron $34: 13-25$

64. Ibanez CF, Simi A (2012) p75 neurotrophin receptor signaling in nervous system injury and degeneration: paradox and opportunity. Trends Neurosci 35(7):431-40

65. Woo NH et al (2005) Activation of p75NTR by proBDNF facilitates hippocampal long-term depression. Nat Neurosci 8(8): 1069-77

66. Lu B (2003) Pro-region of neurotrophins: role in synaptic modulation. Neuron 39(5):735-8

67. Lee R et al (2001) Regulation of cell survival by secreted proneurotrophins. Science 294(5548):1945-8

68. Rosch $\mathrm{H}$ et al (2005) The neurotrophin receptor p75NTR modulates long-term depression and regulates the expression of AMPA receptor subunits in the hippocampus. Proc Natl Acad Sci U S A 102(20):7362-7

69. Rodrigues SM, LeDoux JE, Sapolsky RM (2009) The influence of stress hormones on fear circuitry. Annu Rev Neurosci 32:289-313

70. Anagnostaras SG, Gale GD, Fanselow MS (2001) Hippocampus and contextual fear conditioning: recent controversies and advances. Hippocampus 11(1):8-17

71. Goshen I et al (2011) Dynamics of retrieval strategies for remote memories. Cell 147(3):678-89 